\title{
Globalización, soberanía y patrimonio alimentario
}

Luis Páez*

Resumen

LA GLOBALIZACIÓN ES UN FENÓMENO EN DONDE LA HOMOGENIZACIÓN TIENDE A SER RECURRENTE. UNA HOMOGENIZACIÓN NO SÓLO ECONÓMICA O POLÍtICA, SINO TAMBIÉN CULTURAL. DESDE ESTE PUNTO DE VISTA, LA GLOBALIZACIÓN ECONÓMICA Y CULTURAL EN RELACIÓN A LA PRODUCCIÓN, TRANSFORMACIÓN, DISTRIBUCIÓN Y CONSUMO DE LOS ALIMENTOS CONLLEVA ESPECIAL INTERÉS, YA QUE NO SÓLO SE TRATA DE LA PÉRDIDA DE LA DIVERSIDAD CULTURAL, -ENTENDIDA EN ESTE CASO COMO EL CÚMULO DE CONOCIMIENTOS EN RELACIÓN A LAS PRÁCTICAS AGROALIMENTARIAS-, SINO TAMBIÉN DE LA PÉRDIDA de la agrobiodiversidad en el planeta. Frente a este fenómeno, desde la agricultura familiar campesina, NACE EL CONCEPTO DE SOBERANía ALIMENTARIA, EL CUAL BUSCA FRENAR LA HOMOGENIZACIÓN PROVOCADA POR LA AGROINDUSTRIA, PRINCIPAL CARACTERÍSTICA DE LA GLOBALIZACIÓN ALIMENTARIA. ASIMISMO, SURGE EL CONCEPTO DE PATRIMONIO ALIMENTARIO, COMO UNA PROPUESTA COMPLEMENTARIA AL DE LA SOBERANÍA ALIMENTARIA, BASADA EN EL vínculo entre patrimonio natural y patrimonio cultural en torno a la alimentación. Por lo tanto, ambas PROPUESTAS BUSCAN PROMOVER TANTO LA DIVERSIDAD CULTURAL COMO LA AGROBIODIVERSIDAD DEL PLANETA, LAS CUALES SE ENCUENTRAN AMENAZADAS POR LOS EFECTOS DE LA GLOBALIZACIÓN.

Palabras Clave: globalización - SOberanía alimentaria - patrimonio alimentario - agricultura familiar CAMPESINA - AGROINDUSTRIA.

Abstract

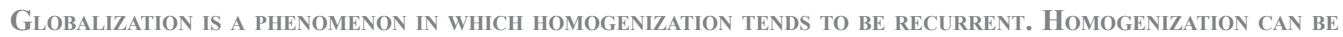
CULTURAL AS WELl aS ECONOMiC OR POLITICAL. From this POINT OF VIEW, ECONOMiC AND CULTURAL GLOBalization IN RELATION TO THE PRODUCTION, PROCESSING, DISTRIBUTION AND CONSUMPTION OF FOOD HAS ADITIONAL RELEVANCE, SINCE IT IS NOT ONLY THE LOSS OF CULTURAL DIVERSITY, -UNDERSTOOD IN THIS CASE AS THE WEALTH OF KNOWLEDGE IN RELATION TO THE PRACTICES AGROALIMENTARY-, BUT ALSO WITH THE LOSS OF THE AGROBIODIVERSITY OF THE PLANET. IN OPOSITION TO THIS PHENOMENON, THE CONCEPT OF FOOD SOVEREIGNTY IS GENERATED BY FAMILY FARMING, WHICH SEEKS TO STOP THE HOMOGENIZATION CAUSED BY AGRIBUSINESS, WHICH IS A MAJOR CHARACTERISTIC OF FOOD GLOBALIZATION. IN ADDITION, THE CONCEPT OF FOOD HERITAGE ARISES A COMPLEMENT TO FOOD SOVEREIGNTY, CORRESPONDING TO THE LINK BETWEEN NATURAL HERITAGE AND CULTURAL HERITAGE AS THEY RELEATED TO NUTRITION. THEREFORE, BOTH PROPOSALS SEEK TO PROMOTE BOTH THE CULTURAL DIVERSITY AND AGROBIODIVERSITY OF THE PLANET, WHICH ARE THREATENED BY THE EFFECTS OF GLOBALIZATION.

KEYWORDS: GLOBALIZATION - FOOD SOVEREIGNTY - FOOD HERITAGE - FAMILY FARMING - AGRO-INDUSTRY.

* Antropólogo, especialista universitario en Planificación, Cooperación y Desarrollo. Diplomado en Cooperación Cultural. Magister en Estudios Latinoamericanos Correo electrónico: luis.paezv@gmail.com 


\section{Introducción}

El actual sistema global alimentario promueve una constante y paulatina pérdida de agrobiodiversidad, así como también de las diversas prácticas culturales asociadas a la alimentación. Asimismo, los impactos de la agroindustria por sobre la sociedad se acrecientan cuando éste modelo provoca, al mismo tiempo, hambre, desnutrición y obesidad en el planeta, generando así, una grave situación alimentaria.

Sin embargo, el fenómeno agroalimentario cobra especial relevancia cuando, a pesar de la los embates de la globalización, millones de personas dedicadas a la agricultura familiar campesina y a la salvaguardia de sus prácticas culinarias, se transforman en verdaderos guardianes de su patrimonio alimentario, promoviendo de este modo la soberanía alimentaria, la agrobiodiversidad y la diversidad cultural.

En el presente artículo veremos la importancia de la cultura dentro del análisis del fenómeno agroalimentario, así como también algunas de las respuestas "culturales" que se generan para hacer frente a un proceso de homogenización promovido por el actual sistema de globalización alimentaria.

\section{Antropología, alimento y poder}

La temática alimentaria puede ser abordada desde distintas ópticas y disciplinas tales como la ecología, la nutrición, la economía y la antropología por poner algunos ejemplos; por lo que no es difícil asumir que la alimentación se encuentra atravesada por múltiples y distintos elementos que de una $u$ otra manera afectan a las personas. Y es que el hecho de alimentarnos representa una de las acciones más importantes en la vida de un individuo y por ende de la sociedad humana, puesto que permite nuestra reproducción biológica y social y con ello la continuidad y permanencia de nuestra especie.

Debido a las características teóricas y metodológicas propias de la Antropología sociocultural, esta disciplina científica ofrece una mirada integral del fenómeno alimentario. No obstante, dentro de sí misma existen diversos abordajes sobre la alimentación, ya que el estudio de las prácticas alimentarias -ya sea desde sus aspectos materiales, sociales o simbólicos- se constituyen en un mecanismo de análisis que incluye también a otros espectros de la sociedad (Gracia, n/d). En este sentido,

“...la producción, distribución y consumo de alimentos ha estado en la base de numerosos estudios de antropología económica y ecológica; la selección cuidadosa de alimentos, la ofrenda sagrada de manjares, las interdicciones o las preferencias que recaen sobre ciertas comidas y personas han llenado capítulos de trabajos sobre religión y sistemas de creencias; la comida como medio para el establecimiento derechos y obligaciones entre familiares, parientes o amigos o como forma de manifestar agradecimiento, compromiso, deuda o cariño han sido temas tratados por la antropología del parentesco; del mismo modo, el vocabulario alimentario, así como los campos semánticos o las taxonomías culinarias han sido atendidos desde la antropología cognitiva...,la ritualización y las fiestas, la comensalidad, los recetarios, los calendarios de actividades y comidas, los productos de la tierra, la restitución o la invención de nuevos formas de abastecer los mercados ha llamado, a su vez, la atención de los estudiosos del patrimonio cultural y de los estudios regionales; del mismo modo, los alimentos como fuente de salud y enfermedad, de males y remedios, han tenido un lugar también en los trabajos de antropología de la medicina (Ibíd.: 1) [énfasis propio].

Por lo tanto, podemos observar que las distintas ramas de la antropología han procurado el abordaje de una temática específica relacionada con la alimentación, ya sea desde un punto de vista social, económico, ritual o médico, según el interés del objeto de estudio. Sin embargo, si hablamos de integralidad es importante dimensionar al fenómeno alimentario no por separado sino en su conjunto, con todos los aspectos que le rodean. 
Para ello, recurrimos a la propuesta de Marcel Maus, quien habla de que la sociedad tiene que verse como un hecho social total, en el que todas las áreas de la cultura y los distintos tipos de instituciones (políticas, económicas, religiosas, legales, etc.) se expresan simultáneamente e influyen en toda la sociedad (Ibíd.). Es así, que analizar el hecho alimentario puede revelar la estructura de un orden social establecido, es decir, de un sistema alimentario local, nacional e incluso global, el mismo que:

\begin{abstract}
“depende de y afecta al resto de sistemas -económico, político, familiar, cultural- que están articulando cada realidad social, de forma que es imposible analizar la cultura alimentaria sin vincularlo, como apunta Goody (1982), con el reparto de poder y autoridad dentro de la esfera económica y política y, en consecuencia, también con el sistema de estratificación social y la división sexual y social del trabajo" (Ibíd.: 2).
\end{abstract}

Con esta perspectiva integral "nace" la disciplina específica de la antropología de la alimentación, la misma que busca entender el fenómeno alimentario desde todas sus aristas, reconociendo así "los tratamientos científico nutricionales, políticos y económicos específicos y autónomos, y propone un estudio interrelacionado de dichas facetas para acceder a conocer la naturaleza del fenómeno y sus implicaciones" (Carrasco, 2007: 83). Además, es importante anotar que la antropología de la alimentación también se encuentra ligada a la Antropología aplicada, con el análisis de las políticas alimentarias, y con el uso "político" de las mismas.

Inicialmente, a más del conocimiento científico que la antropología pretendía generar, esta disciplina fue utilizada como herramienta de colonización en la que el estudio alimentario también jugaba un rol preponderante. Tal es el caso de la creación de organismos como el Instituto Internacional de África, que en 1934 crea un comité en el que antropólogos y nutricionistas estudian la dieta de las culturas de dicho continente. Asimismo, "el uso de las colonias como "productoras de cultivos rentables" promovió el desarrollo industrial del imperio y tuvo como efecto la transformación radical de los sistemas alimentarios y del medioambiente colonizado" (Ibíd.:87). Tras la segunda guerra mundial los nuevos organismos internacionales releyeron a la antropología aplicada como una herramienta para "sacar" del "subdesarrollo" a sociedades y países del llamado "tercer mundo", es decir, sin un cambio sustancial en su concepción en concordancia con las relaciones de poder. Por ello, consideramos válido el precepto de Carrasco, quien llama

\footnotetext{
"a entender los problemas alimentarios como el resultado de la configuración de una estructura que somete a la población afectada a nuevas condiciones biológicas (desnutridos o mal nutridos) y nuevas condiciones sociales (dependientes, ayudados o subsidiados en la dimensión más básica del ser humano)" (Ibíd.: 94).
}

En consecuencia, y para el caso que aquí nos convoca, el fenómeno alimentario global será visto desde las relaciones de poder generadas a partir de un sistema mundo alimentario basado en un modelo de agroindustria y agronegocio, tal y como revisaremos en el siguiente apartado.

\title{
La globalización alimentaria
}

Si tomamos a la antropología de la alimentación como una disciplina que mira el hecho total alimentario, en donde se revela un orden social establecido, en el que existen relaciones de poder y en el que se afectan las condiciones biológicas, nutricionales, económicas, culturales y sociales de la población, resulta necesario entonces reconocer los impactos que el actual sistema alimentario global promulga.

Dichos impactos evidencian un mundo en donde en la actualidad existen alrededor de 870 millones de personas que padecen de hambre (de las cuales 52 se encuentran en América Latina, región rica en agrobiodiversidad) (FAOa, 2012), en donde existen altos índices de 
desnutrición, subnutrición, sobrepeso y obesidad de manera simultánea, en donde las sociedades de abundancia desperdician grandes cantidades de comida (FAO, 2012b), en donde el proceso de industrialización ha modificado nuestra forma de producir, comerciar y consumir los alimentos, y en donde la globalización ha provocado tanto una pérdida paulatina de la agrobiodiversidad mundial, como una tendencia a la homogenización cultural alimentaria.

En relación a la pérdida de agrobiodiversidad los datos son preocupantes, ya que:

\begin{abstract}
“A pesar de la importancia vital que tiene la agrobiodiversidad para la supervivencia humana, está desapareciendo a un ritmo cada vez mayor. Se estima que, a lo largo de la historia de la humanidad, se han utilizado unas 10.000 especies para la alimentación humana y la agricultura. Actualmente, tan sólo 12 especies vegetales y cinco especies animales nos proporcionan más de un $70 \%$ de nuestros alimentos. Solamente cuatro especies vegetales (papa, arroz, maíz y trigo) y tres especies animales (vacas, cerdos y pollos) nos proporcionan más de la mitad” (Tapia, 2011: 25).
\end{abstract}

Por su parte, la homogenización cultural alimentaria se evidencia principalmente en las grandes cadenas de supermercados. Por ejemplo,

\begin{abstract}
"Si usted entra al Supermaxi para hacer las compras entra al mismo tiempo al mundo agroalimentario globalizado: el yogur puede ser de una empresa de origen francés: Danone, la leche de una multinacional con sede en Suiza: Nestlé, los bizcochos salados Ritz están fabricados por la transnacional americana Nabisco. En este sentido hace poca diferencia, si usted hace sus compras en Quito o en Bogotá, si se va a un supermercado en Bangkok o en Johannesburgo: las marcas de las grandes empresas internacionales están presentes y dominan en todos los rincones del mundo" (Hidalgo, en Brassel, Breilh, y Zapata, 2011: 7).
\end{abstract}

Es importante anotar que el impacto que tiene la globalización en el actual sistema alimentario predominante, no se debe en sí mismo al fenómeno de la globalización, ya que de por sí "los alimentos y la globalización son inseparables. Desde la antigüedad el comercio a larga distancia ha involucrado a los alimentos básicos y productos de lujo, como el vino, té, café, arroz, especias y pescado seco" (Nützenadel y Trentmann, 2008: 1)1 [Traducción propia].

En consecuencia, el origen del actual sistema alimentario global y de sus diversos impactos es relativamente reciente, ya que se debe al desarrollo de un modelo agrario basado en la denominada revolución verde, implementada en el mundo entero a partir de la década de 1960. La revolución verde se basa en el fomento de la agricultura industrial a través de paquetes tecnológicos que incluyen semillas híbridas, fertilizantes, plaguicidas, maquinaria agrícola y sistemas de riego. Su prioridad es elevar los niveles de productividad y rentabilidad agrícola para lo cual promociona el monocultivo intensivo (Bustos, 2010).

La aplicación de la revolución verde fue "eficaz" en cuanto al incremento de la producción de alimentos en el mundo, ya que en tan sólo medio siglo dicha producción se duplicó. "En 1950, la tierra contaba con alrededor de 2.500 millones de individuos, disponiendo de un promedio de 2.450 kilocalorías por día; para el año 2000, fueron 6.000 millones de habitantes que dispusieron, en promedio, de 2.700 kilocalorías" (Ibíd.: 14).

No obstante, tal y como vimos en párrafos anteriores, las implicaciones de la revolución verde tienen varias críticas a su haber, ya que pesar del incremento agroproductivo, el hambre, la desnutrición y la malnutrición continúan siendo un problema que aqueja a millones de personas en el mundo (aumentar la producción no necesariamente implica una adecuada redistribución). Asimismo, la homogenización cultural alimentaria y la pérdida de la agrobiodiversidad son la consecuencia de este modelo agrario, el cual, si bien es predominante, ha encontrado múltiples resistencias, entre las que se encuentran las propuestas de soberanía alimentaria y de patrimonio alimentario.

1 "Since ancient times long-distance trade has involved staple foods and luxury products such as wine, tea, coffee, rice, spices and dried fish" (texto original). 


\section{La soberanía alimentaria}

El concepto de soberanía alimentaria fue elaborado por uno de los movimientos sociales más grandes del mundo: La Vía Campesina. Este concepto nace como una contrapropuesta al concepto de seguridad alimentaria ${ }^{2}$ en el foro mundial de organizaciones sociales y no gubernamentales de 1996, evento paralelo al de la Cumbre Mundial sobre la Alimentación que buscaba tratar el tema desde sus propias experiencias, planteamientos y puntos de vista (Vallejo, 2008). Por lo tanto, se trata de un concepto que surgió desde los propios campesinos y movimientos sociales de la época, quienes manifestaron que:

"Soberanía alimentaria es el derecho de cada nación para mantener y desarrollar su propia capacidad para producir los alimentos básicos de los pueblos, respetando la diversidad productiva y cultural. Tenemos el derecho a producir nuestros propios alimentos en nuestro propio territorio de manera autónoma. La soberanía alimentaria es una precondición para la seguridad alimentaria genuina" (Bravo, 2009: 78-79).

Este concepto fue evolucionando con el pasar de los años, y hoy se lo concibe como:

"El derecho de los pueblos a alimentos sanos y culturalmente adecuados, producidos mediante métodos sostenibles, así como su derecho a definir sus propios sistemas agrícolas y alimentarios. Desarrolla un modelo de producción campesina sostenible que favorece a las comunidades y su medio ambiente. Sitúa las aspiraciones, necesidades y formas de vida de aquellos que producen, distribuyen y consumen los alimentos en el centro de los sistemas alimentarios y de las políticas alimentarias, por delante de las demandas de mercados y empresas. La soberanía alimentaria da prioridad a la producción y consumo local de alimentos. Proporciona a un país el derecho de proteger a sus productores locales de las importaciones baratas y controlar la producción. Garantiza que los derechos de uso y gestión de tierras, territorios, agua, semillas, ganado y biodiversidad estén en manos de quien produce alimentos y no del sector empresarial. Así, la implementación de una auténtica reforma agraria constituye una de las prioridades del movimiento campesino. La soberanía alimentaria se presenta hoy en día como una de las repuestas más potentes a las actuales crisis alimentaria, de pobreza y climática" (Vía Campesina, 2015,s/p).

Si bien no se trata de un concepto terminado, la importancia de la soberanía alimentaria radica en sentar principios específicos y claros en defensa de la agricultura campesina como principal fuente de seguridad alimentaria (Bravo, 2009). Desde este punto de vista, se destaca el interés por la agrobiodiversidad y por las diversas prácticas y saberes en torno a la producción agrícola, así como también a las múltiples formas de alimentarnos, las políticas alimentarias nacionales, y el acceso a los recursos agua, tierra y semillas principalmente.

La sociedad civil organizada que impulsa la implementación de la soberanía alimentaria a lo largo y ancho del planeta, cobra especial relevancia dentro de las luchas por los derechos de la alimentación ya que "al confrontar la globalización neoliberal y la globalidad imperial, los movimientos locales, nacionales y transnacionales pueden ser vistos como una forma de globalización contra-hegemónica” (Escobar, 2013: 71).

En consecuencia, la soberanía alimentaria se constituye como un mecanismo de defensa en un escenario de creciente globalización, en el que campesinos y campesinas de todo el mundo buscan desde un enfoque de derechos, no sólo el acceso al alimento sino también a una producción soberana del mismo, así como también una alimentación sana, con identidad y culturalmente apropiada³.

2 El concepto de seguridad alimentaria, propuesto por la FAO, contempla cuatro aspectos fundamentales que son: a) disponibilidad de alimentos (se refiere a la cantidad de alimentos disponibles), b) acceso a los alimentos (posibilidad de adquisición de alimentos), c) estabilidad (abastecimiento y acceso continuo de alimentos), y d) uso o utilización biológica de los alimentos (prácticas alimenticias adecuadas y la capacidad de absorción de nutrientes) (Calero, 2011).

3 Es importante mencionar que en todo el mundo, sólo Bolivia y Ecuador han acogido de manera oficial el concepto de Soberanía Alimentaria a través de sus respectivas Constituciones Políticas. 


\section{El patrimonio alimentario}

La idea de patrimonio cultural surge en Europa después de la segunda guerra mundial, al constatar que la guerra vulneró múltiples obras y monumentos de gran riqueza cultural para las sociedades de mencionada región, las cuales fueron consideradas como herencia y legado para las nuevas generaciones. Desde esta perspectiva, la idea de patrimonio nace, en primera instancia, como un sentido de herencia y tradición, y en segundo lugar, como una visión fuertemente ligada a lo material y monumental (obras de arte y arquitectónicas son ejemplo de ello).

Con el paso del tiempo el concepto de patrimonio cultural amplió su espectro, y hoy en día la UNESCO lo considera como: "El conjunto de bienes que caracterizan la creatividad de un pueblo y que distinguen a las sociedades y grupos sociales unos de otros, dándoles su sentido de identidad, sean estos heredados o de producción reciente" (UNESCO, 1972). Por lo tanto, se aprecia que el patrimonio ya no sólo hace referencia a la herencia y a la tradición, ni tampoco solamente a los bienes materiales, sino también a la creatividad, a la innovación y a las expresiones culturales inmateriales, tales como la danza, las manifestaciones religiosas, el lenguaje, e incluso la alimentación. A decir de Juárez,

"El valor cultural del acto de comer es cada vez más entendido como un acto patrimonial, pues la comida es traductora de pueblos, naciones, civilizaciones, grupos étnicos, comunidades, familias, personas. El sentido de pertenencia a una sociedad y a una cultura, nace primordialmente por hablar un idioma, compartir recetas y platos, crear hábitos cotidianos de la comida. Entonces la comida es también un lugar que define y confiere a la persona su pertenencia; es decir, tener una identidad..." (2008: 119).

En este sentido, al ser la alimentación humana parte de las prácticas culturales e identitarias de las sociedades, es completamente plausible considerarla como un patrimonio. No obstante, lo interesante de ello, es que no solamente se la puede considerar como un patrimonio cultural, sino también como un patrimonio natural.

"Con esta reflexión, estamos apuntando a la enorme importancia que tiene la disponibilidad de productos agropecuarios o acuícolas como soporte del arte de la cocina y como medios de expresión usados por ésta. Basta imaginar qué catástrofe, ya no solo biológica sino cultural, sería la desaparición del maíz en América" (Merino en Unigarro, 2010: 16).

Asimismo, las prácticas agrícolas también pueden ser consideradas como patrimonio de los pueblos. Para el efecto, la FAO desarrolló un concepto conocido como SIPAM (Sistemas Ingeniosos de Patrimonio Agrícola Mundial), el cual busca identificar sistemas agrícolas excepcionales que contribuyan a un mejor manejo de los agroecosistemas. Bajo esta perspectiva, los SIPAM son definidos como un sistema vivo, en evolución, de comunidades humanas en una intrincada relación con su territorio, ambiente cultural o agrícola o ambiente biofísico y social más amplio (http:// www.fao.org/giahs/giahs/concepto-de-patrimonio-agricola/es/). Su importancia radica en que:

“...los sistemas SIPAM se ha desarrollado y adaptado para enfrentar diversas situaciones de cambio como eventos naturales, tecnologías nuevas o cambios políticos y sociales, con el fin de aliviar posibles riesgos y garantizar la seguridad alimentaria y la supervivencia de sus habitantes. Las estrategias dinámicas de manejo y los procesos que permiten el mantenimiento de la biodiversidad y de los servicios esenciales de estos ecosistemas se caracterizan por una continua innovación tecnológica y cultural y por la transferencia de conocimientos entre generaciones y el intercambio con otras comunidades y ecosistemas. La riqueza y amplitud del conocimiento y de las experiencias atesoradas en el manejo y en el uso de los recursos naturales son elementos de importancia global que necesitan ser preservados, y al mismo tiempo, evolucionar para subsistir" (Ibíd.) ${ }^{4}$.

4 Es importante destacar que en el Ecuador la FAO y el Ministerio de Cultura y Patrimonio han lanzado un proceso interesante de identificación de sistemas de patrimonio agrobiodiverso, identificando 7 sistemas en todo el territorio ecuatoriano. Éstos son: 1) la chakra andina, 2) el wachu rosado y las fincas pastusas, 3) las huertas paltas y las siembras de agua, 4) la aja shuar, 5) la chakra amazónica, 6) la finca montubia, y 7) los pueblos de manglar (MCyP-FAO, 2014). Para mayor información sobre este tema revisar: http:/www.allpachaski.com/2015/07/agricultura-ancestral-en-el-ecuador/ 
En este sentido, patrimonio alimentario significa todo aquel alimento con importancia simbólica, cultural e identitaria que tiene para un país o territorio determinado. El alimento patrimonial no sólo hace relación a la preparación de platos o bebidas tradicionales, sino también a los productos que se utilizan como ingredientes. Por ello, hablamos de patrimonio cultural y natural alimentario. A modo de ejemplo, podríamos señalar que la colada morada, por su valor identitario para el Ecuador, sería considerada como un alimento de patrimonio cultural, mientras que el mortiño - principal ingrediente de la colada morada - sería considerado como un producto de patrimonio natural.

Asimismo, la cocina se convierte en un espacio fundamental en donde el patrimonio alimentario se recrea, y en el que los saberes y los sabores se conjugan para crear una variedad infinita de platos y bebidas que, a pesar de los embates de la globalización, tenemos a nuestra disposición. De este modo,

"La cocina, como espacio integrador, permite una experiencia en la que dialogan y se enfrentan cosmovisiones, lugares de enunciación, estructuras de poder, etc., que no aparecen de manera accidental sino que se desprenden del mismo hecho de ser una expresión del ser social. La distribución de roles, no sólo en la producción de los alimentos, sino también en su preparación o consumo; las significaciones atribuidas como tabúes, prohibiciones y preferencias individuales y colectivas; cualidades medicinales o de enfermedad; temporalidades ligadas, como es el caso específico de comidas ofrecidas en épocas de fiesta o celebraciones religiosas; lugares apropiados e inapropiados para comer; diferenciaciones socioeconómicas y de roles; formas de servir los platos; selección de alimentos con que se acompañan ciertos preparaciones, por nombrar sólo algunos, hablan de una serie de elementos de carácter ideológico, que permiten dilucidar un entramado de dinámicas que se surten en torno a la cocina y que manifiestan creencias, relaciones der poder, prácticas que no se reducen a la fugacidad de una preparación o el consumo de un plato (Unigarro, 2010: 85).

Desde este punto de vista resulta necesario considerar a la alimentación como un fuerte marcador étnico de las sociedades, y como uno de los elementos que contribuyen a generar identidad mediante la constatación de la diferencia y la promoción de la diversidad cultural (Ochoa, 2009).

\section{Conclusiones}

El actual sistema global alimentario promueve tanto la homogenización agroproductiva como la homogenización cultural. En este sentido, los conocimientos agrícolas, las técnicas culinarias, las dietas, los saberes y los sabores se han visto alterados. De este modo, la imposición de una cultura alimentaria basada en agronegocio y en el fast food ${ }^{5}$ ha provocado múltiples impactos que van desde el detrimento de la salud humana, hasta el cambio de hábitos alimenticios y prácticas culturales entorno a los mismos.

En este contexto, han surgido múltiples respuestas que impulsan la soberanía alimentaria y que a su vez promueven y revalorizan el patrimonio alimentario, el cual cumple un rol significativo para la salvaguardia de prácticas alimentarias sanas, sostenibles y culturalmente apropiadas, es decir, una alimentación y una nutrición con identidad, basada en la producción local, en el entorno familiar de la mesa y de la cocina, en la esfera de lo cotidiano, lo ritual y lo festivo de una población; y de este modo alimentar tanto el cuerpo como el alma.

Por lo tanto, la cultura, como parte integral del hecho total alimentario, se convierte también en un mecanismo de defensa y en una herramienta de lucha de campesino/as y cocinero/as que desde sus prácticas y conocimientos acumulados, plantean alternativas políticas en respuesta a este fenómeno de globalización. En tal sentido, la dimensión cultural del alimento cobra especial relevancia para entender, no sólo las prácticas culturales asociadas a la agroalimentación, sino también acerca de las prácticas políticas ligadas a este tema.

5 Fast food es un término anglosajón que se utiliza para denominar a todas aquellas comidas que se preparan de manera rápida e inmediata. 


\section{Bibliografía}

Bustos, Blanca. 2010, "Hacia la soberanía alimentaria”, en: Hortencia Bustos (comp.) Hacia la soberanía alimentaria agroecología y comercio asociativo desde experiencias Andino-Amazónicas, Ediciones La Tierra -UASB - GTZ. Quito.

Bravo, Ana Lucía. 2009, Análisis de las políticas agrícolas aplicadas en el Ecuador en los noventas desde la perspectiva de la soberanía alimentaria, tesis para obtener el título de maestría en ciencias sociales con mención en estudios socioambientales, FLACSO, Quito.

Calero, Carla. 2011, Seguridad alimentaria en Ecuador desde un enfoque de acceso a alimentos, Editorial ABYAYALA, FLACSO, Quito.

Carrasco, Noelia. 2007, Desarrollos de la antropología de la alimentación en América Latina: hacia el estudio de los problemas alimentarios contemporáneos, Centro de Investigación en Alimentación y Desarrollo, Chile.

Escobar, Arturo. 2013, Una minga para el postdesarrollo: lugar, medio ambiente y movimientos sociales, Ediciones Desde Abajo, Bogotá.

FAO (a). 2012, El estado de la inseguridad alimentaria en el mundo, Roma, 2012 (edición electrónica).

FAO (b). 2012, Pérdida y desperdicios alimentarios en el mundo, Roma, 2012 (edición electrónica).

1996, Cumbre mundial sobre la alimentación, Roma, (edición electrónica).

Gracia, Mabel. (e.p.): Los enfoques teóricos en antropología de la alimentación, edición electrónica, s. 1., s.f., (edición electrónica).

Hidalgo, Francisco. 2011, Introducción. en: Frank Brassel, Jaime Breilh, y Alex Zapata, (Eds.) ¿Agroindustria y soberanía alimentaria? Hacia una ley de agroindustria y empleo agrícola, SIPAE, Quito.

http://www.fao.org/giahs/giahs/concepto-de-patrimonio-agricola/es/ (ingresado el 20 de octubre de 2015).

http://www.viacampesina.org/es (ingresado el 19 de marzo de 2015).

Juárez, J.L. 2008, "El boom culinario mexicano", en: Nacionalismo culinario. La cocina mexicana del siglo XX, Consejo nacional para la Cultura y las Artes, México.

MCyP y FAO. 2014, Apoyo a la identificación y conservación de los sistemas ingeniosos del patrimonio agrícola nacional. Segunda Fase, Documento Inédito, Agosto.

Merino Medina Augusto. 2010, "Introducción: Marco teórico para el estudio de la cocina popular tradicional en Iberoamérica”, en: Unigarro, Catalina, Patrimonio cultural alimentario, Fondo Editorial Ministerio de Cultura-CAB-IPANC-MC, Quito.

Nützenadel Alexander and Frank Trentmann. 2008, "Mapping Food and Globalization”, en: Alexander Nützenadel and Frank Trentmann (eds.) FOOD AND GLOBALIZATION, BERG, Nueva York.

Ochoa Roberto y M. Esthet. 2009, Las nuevas culturas alimentarias: globalización vs etnicidad, UPV/EHU, Bilbao (edición electrónica).

UNESCO. 1972, Convención Mundial sobre Protección del Patrimonio Cultural y Natural, (edición electrónica). Unigarro, Catalina. 2010, Patrimonio cultural alimentario, Fondo Editorial Ministerio de Cultura -CAB-IPANC- MC, Quito.

Vallejo Silvana. 2008, "La seguridad y la soberanía alimentaria en el marco de la globalización”, en: Almeida, Patricio, Seguridad alimentaria, pobreza y desarrollo, revista de análisis económicos, Quito. 